\title{
AFRONTAMIENTO FAMILIAR ANTE LA ENFERMEDAD MENTAL
}

\author{
Ana García Laborda* \\ J. Carlos Rodríguez Rodríguez** \\ *Enfermera en Salud Mental. Socióloga. Antropóloga \\ **Sociólogo Antropólogo
}

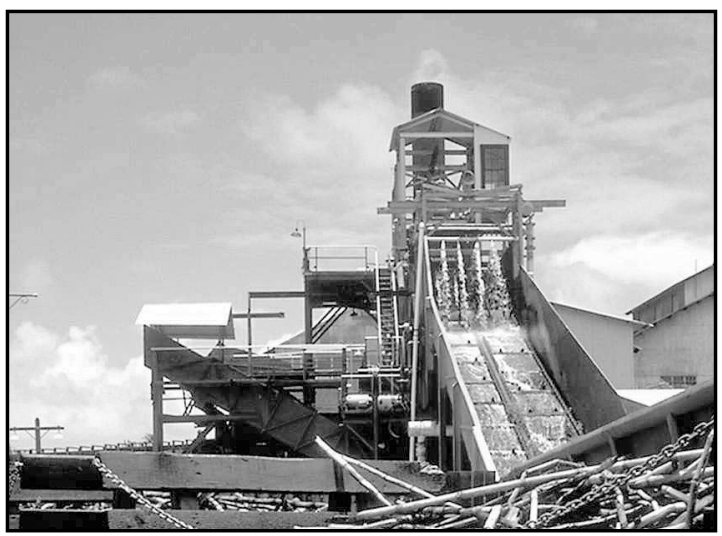

FAMILY COPING WITH MENTAL ILLNESS

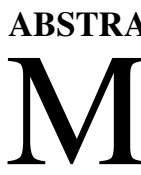
change according to resources used. Amongst the resources, the main factor is the family, thus the relevance of the family support network for the management of the person with mental health problems

Key words: Coping. Behaviour towards disease. Family. Mental illness.

\section{RESUMEN}

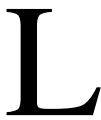
a enfermedad mental tiene implicaciones tanto personales como familiares. Tanto los estilos de afrontamiento como la conducta de enfermedad varían en función de los recursos que el sujeto pone en juego, entre los recursos ambientales el principal es la familia, de aquí la especial importancia que cobra el grupo familiar en el tratamiento de la persona con enfermedad mental.
PALABRAS CLAVES: Afrontamiento. Conducta de enfermedad. Familia. Enfermedad mental.

【 as distintas formas de asumir realidades dolorosas como son la enfermedad y la muerte forman parte de los distintos sistemas culturales, de la filosofía y de los mensajes de las religiones. El sentido del dolor y de lo irreparable son cuestiones cuyo planteamiento y resolución son determinados por los posibles modos de pensar o de creer. En este plano del pensamiento o de la creencia se pone de manifiesto la enorme dimensión existencial que adquiere, para cada persona, el modo de afrontar tanto la enfermedad como la muerte.

Los modos de conducirse cada cultura con respecto a las crisis vitales se encuentran fundamentados en el sistema social y el modo en que las personas reciben apoyo de sus relaciones personales. Las creencias con respecto a la enfermedad mental se configuran respecto a los distintos modos, hábitos y las costumbres de las personas. Los modos culturales entendidos como costumbres adquiridas a lo largo de siglos y que dan sentido a las dificultades y tragedias de la vida establecen comportamientos y sirven de ayuda para que los individuos implicados encajen el estrés utilizando un modo de conducta especifica y reactiven apoyos familiares, sociales y religiosos que facilitan adaptación psicosocial a la enfermedad mental.

Por tanto, es necesario tener en cuenta las implicaciones culturales, sociales, económicas, familiares, etc., que la enfermedad tiene para cada individuo. Estos y las familias desarrollan conductas adaptativas, así como una forma de expresar las nuevas relaciones, que se establecen entre el enfermo mental y los miembros de la familia, ya sea para modificarlas, para legitimizar un cambio de rol o para asumir el rol de enfermo mental. 


\section{AFRONTAMIENTO Y CONDUCTA DE ENFERMEDAD}

El concepto de afrontamiento hace referencia al conjunto de esfuerzos cognitivos y conductuales, permanentemente cambiantes, desarrollados para hacer frente a las demandas específicas, externas o internas, evaluadas como abrumadoras o desbordantes de los propios recursos.

$\mathrm{Si}$ estos esfuerzos resultan efectivos en la resolución de los problemas proporcionarán alivio, recompensa, tranquilidad y equilibrio: en definitiva, disminuirán el estrés. La familia debe evaluar la situación enfrentándose a la enfermedad redefiniendo sus roles, y modificando sus metas a corto o largo plazo, asumiendo la alteración, temporal o no, de sus relaciones con el enfermo, adoptando una actitud práctica, flexible y aprendiendo a detectar y resolver los problemas, según se presenten en la vida y en el desarrollo de la enfermedad del miembro enfermo de la familia. Existen en cambio, otras actitudes que impiden afrontar debidamente las dificultades, y aparecen en los sujetos que niegan sistemáticamente todos los problemas o en aquellos que sintiéndose abrumados por su "mala suerte", transfieren todas sus responsabilidades a otros.

El afrontamiento ha sido estudiado tanto en las investigaciones biologicistas realizadas sobre el estrés en los años 40-50, como en el psicoanálisis de Freud (modelo psicoanalítico), que introdujo la cuestión de los mecanismos de defensa del yo, según quedaban establecidos en la dinámica inconsciente de la personalidad.

Los sociólogos también han analizado la situación de "encontrarse enfermo" en su dimensión social propugnando el modelo mediante el cual la sociedad provee de un rol especifico, el rol de enfermo a las personas sometidas a tratamiento o asumidas por las instancias asistenciales. Concretamente Parsons entiende que son características de este rol: la exención de las responsabilidades sociales normales, un cierto derecho a recibir apoyo, ayuda o tratamiento y la suposición de que el sujeto quiere curarse y pone los medios para conseguirlo.

El término conducta de enfermedad fue definido más tarde por Mechanic refiriéndose concretamente a los aspectos sociales del "estar enfermo"; es decir, los distintos modos en que los síntomas del enfermar serían percibidos, evaluados y vividos por los diferentes individuos, lo cual estaría en relación con la existencia de una serie de normas y valores e incluso, con la posible valoración de la enfermedad como una forma de obtener recompensas o castigos. Es un concepto que sirve para conocer y medir los efectos sociales del proceso del enfermar.

Esta conducta de enfermedad incluye el buscar ayuda sanitaria y tomar las medidas necesarias para combatir los trastornos, buscando apoyo en familiares y amigos. En esta búsqueda de ayuda influirá la frecuencia con la que se presenta la enfermedad en el grupo social al que pertenece el individuo enfermo y la valoración que dicho grupo hace de las manifestaciones del trastorno. La conducta de enfermar tiene tres funciones fundamentales:

- Hacer frente a las demandas sociales y del entorno.

- Crear el grado de motivación necesario para hacer frente a tales demandas.

- Mantener un estado de equilibrio psicológico para dirigir la energía y los recursos hacia las demandas externas.

Se ha hablado también de los componentes a considerar en el proceso de afrontamiento: estilo estructural, conducta adaptativa automática, control del entorno y resultados obtenidos.

El estilo estructural es una característica estable de la personalidad que actúa como predisponente para afrontar de una forma u otra los acontecimientos de la vida. Sin embargo, este estilo tendrán muy poco valor predictivo en los procesos de afrontamiento reales. El afrontamiento según el estilo estructural de la familia sería una dimensión única, siendo así que en realidad el afrontamiento tiene una naturaleza multidimensional. Por ejemplo, los miembros de la familia no solo deben afrontar la enfermedad mental de uno de sus miembros, sino enfrentarse al estrés, la incapacitación, las demandas del personal sanitario y de los tratamientos a los que será sometido, etc., pero al mismo tiempo debe conservar un equilibrio emocional positivo, una autoimagen satisfactoria, y una aceptable relación con el resto de la familia y los amigos; todo esto exige múltiples estrategias de 
afrontamiento de gran complejidad como para ser consideradas unidimensionalmente.

La conducta automática es aquel comportamiento que se traduce en respuestas que no están controladas conscientemente, es decir, que no exigen un esfuerzo. Por ello, estas respuestas no pueden considerarse propiamente como de afrontamiento a pesar de que la mayoría de las conductas de afrontamiento se conviertan en automáticas una vez que son aprendidas. Con respecto al resultado adaptativo, se suele cometer el error de equiparar éste con el afrontamiento, pues mientras que el afrontamiento designa el conjunto de conductas englobadas en él, el resultado adaptativo indica los éxitos conseguidos con tales conductas. El objetivo del fenómeno del afrontamiento sería el de lograr el equilibrio en la adaptación a la situación de enfermedad mental.

Por último, y refiriéndonos al control del entorno, es necesario recordar las múltiples fuentes de estrés que no pueden dominarse, en cuyo caso un afrontamiento eficaz incluye todo aquello que permita al individuo tolerar, minimizar, aceptar o incluso ignorar dichos acontecimientos estresantes que escapan a su control. Existen dos tipos básicos de afrontamiento: el dirigido a resolver el problema, cuando se cree posible que éste sea susceptible de algún cambio y el que pretende equilibrar tanto el duelo por la enfermedad como las nuevas relaciones intrafamiliares, aquel en el que los sujetos intentan cambiar ciertas pautas de conducta, aprender nuevos recursos, etc. De hecho, las personas que creen que el resultado depende en parte de sus propias conductas, afrontan de distinta forma los problemas que las que piensan que todo es fruto del azar, de la casualidad, del destino o de cualquier otra fuerza que escape a su control personal. Los primeros son los que emplean estrategias activas, mientras que los segundos responden fundamentalmente con estrategias defensivas.

Los estilos, modos, conductas o estrategias de afrontamiento pueden considerarse desde un punto de vista afectivo, cognitivo y conductual, aunque es difícil establecer una separación neta debido a las interrelaciones existentes entre ellos dentro del propio sujeto, entre los distintos individuos y entre cada uno de ellos con el entorno. Las estrategias son las maniobras que pone en marcha el sujeto para mantenerse estable ante la enfermedad mental de un miembro de la familia. La interpretación personal que los miembros de la familia hacen de la enfermedad mental respecto a las consecuencias que ésta tenga sobre sí mismo, su familia, actividades, rol social, metas, etc., condiciona respuestas emocionales que pueden incluir: ansiedad, depresión, rabia, resentimiento, vergüenza, culpa, indefensión, desesperanza, euforia, excitación o manía.

\section{ESTILOS DE AFRONTAMIENTO}

Existen dos mecanismos cognitivos básicos, que traducen dos tipos diferentes de actitud: el mecanismo de minimización, asociado a una actitud negativa, y el mecanismo de hipervigilancia que se traduce en una actitud hipocondríaca.

La minimización es la tendencia a la falta de atención, ignorancia, negación o racionalización selectiva de los hechos o del significado de la enfermedad mental y sus consecuencias. La hipervigilancia focalizada en los detalles o puntos más importantes para el propio individuo, se acompaña de rigidez de opinión y de inflexibilidad para adaptarse a lo inesperado, es el mecanismo opuesto al anterior y se da típicamente en el carácter obsesivo, manifestándose como antes señalábamos, en una actitud hipocondríaca. Cuando estos sujetos enferman se muestran compulsivamente atentos a los detalles del tratamiento, y son muy críticos con las demoras en la aplicación de las pautas terapéuticas prescritas por su equipo sanitario. A veces también se muestran dudosos o escépticos sobre el personal que les atiende, exigiendo explicaciones meticulosas sobre los procedimientos diagnósticos y terapéuticos. Sin embargo, estas actitudes irán desapareciendo en la medida en que vayan siendo informados sobre la evolución de la enfermedad, tratamientos aplicados, posibles complicaciones, etc., de forma detallada y cuidadosa.

Refiriéndonos ahora al estilo conductual, hay que decir que la evaluación cognitiva de la amenaza ante cualquier estímulo, incluida la percepción de la enfermedad, genera una reacción adaptativa hacia el propio reto que se basa en raíces biológicas elementales. Podemos distinguir tres tipos de estilo conductual:

- Lucha activa. Abarca desde el esfuerzo por minimizar los posibles efectos de la enferme- 
dad sobre la capacidad funcional, hasta la hiperactividad imprudente que frecuentemente refleja el estrés generado por la debilidad e indefensión que lleva implícita la aceptación del rol de enfermo.

- Capitulación. Se caracteriza por actitudes de pasividad, inactividad y dependencia de los demás. Este tipo de pacientes a menudo provoca disgusto, resentimiento o dejadez en el personal sanitario que los atiende, lo cual a su vez puede condicionar una dependencia más pronunciada y una mayor demanda de servicios por parte del paciente. De algún modo es más importante para él, el que se le preste atención, que las limitaciones producidas por la propia enfermedad.

- Evitación. Se caracteriza por ser una estrategia orientada a liberarse de la obligación implícita de aceptar la enfermedad. Casi siempre se acompaña del tipo cognitivo de minimización, sobre todo como forma de negación. El miedo suele negarse y a veces llega a ser casi imperceptible.

\section{RECURSOS FAMILIARES ANTE LA ENFERMEDAD MENTAL}

Los recursos de los que dispone cada sujeto también van a condicionar el estilo de afrontamiento que éste utiliza. Dichos recursos pueden provenir de él mismo o del ambiente. El individuo dispone de los siguientes tipos de recursos:

- Recursos físicos. Salud y energía física.

- Recursos personales. Son el conjunto de creencias que van a permitirle afrontar las dificultades de forma más satisfactoria. La percepción idiosincrática de cada individuo de su enfermedad condiciona la selección por parte de éste de unas u otras estrategias de afrontamiento.

- Recursos sociales. Son las aptitudes o habilidades que le permiten una mayor o menor comunicación con los demás y con el entorno, de forma adecuada y socialmente efectiva.

- Recursos materiales. Una posición económica favorable aumenta las opciones de afrontamiento, ya que permite un acceso más fácil a la asistencia médica, legal, financiera, etc, con lo que disminuye también en cierto modo la vulnerabilidad del individuo frente a la amenaza.

- Técnicas de resolución de problemas. Se basan en experiencias previas (con la enfermedad u otras situaciones estresantes), información y habilidades cognitivo-intelectuales para aplicar esa información, así como en la capacidad de autocontrol.

- Recursos ambientales. Entre los que se incluyen el marco familiar, amigos, apoyo social, condiciones laborales, tipo de vivienda, etc. Todos estos aspectos tienen una importancia fundamental y van a condicionar de manera importante el tipo de respuesta del individuo, dada la interrelación de éste con el medio que le rodea. Cuanto más adversas o insuficientes sean las condiciones ambientales de un sujeto, tanto más difícil le resultará el hacer frente a cualquier tipo de estrés, incluida la enfermedad, ya que sus recursos de afrontamiento serán más limitados.

\section{INTERVENCIÓN EN LA CRISIS FAMILIAR}

Es preciso definir primeramente lo que se entiende por crisis e intervención en crisis dentro del modelo de psiquiatría comunitaria. La crisis supone la aparición brusca de una sintomatología aguda, generalmente en respuesta a un estrés, que supone una ruptura del ciclo vital del individuo y en la que intervienen factores personales y ambientales. Se desencadena cuando ante un estrés grave, un problema serio o una situación amenazante, los mecanismos habituales del individuo de afrontamiento de problemas y búsqueda de soluciones, no cumplen con sus metas. Se produce un desequilibrio entre la importancia y dificultad del problema, y los recursos de que dispone para enfrentarlo. Puede ocurrir en personas sin patología psiquiátrica o como descompensación de un trastorno mental subyacente. La crisis sería un periodo transicional a partir del cual puede que se desarrolle un trastorno mental porque el individuo se enfrentó al conflicto de forma desadaptada, o al contrario, puede superarla satisfactoriamente, saliendo fortalecido y más capacitado para afrontar futuras dificultades, ya que supo buscar nuevas perspectivas y enfoques.

La noción misma de crisis conlleva la urgencia y la necesidad de una intervención terapéutica. La 
naturaleza de la crisis es muy diversa. Puede surgir como respuesta a un suceso traumático (fallecimiento de un ser querido, separación conyugal, quiebra económica, problemas laborales...); como manifestación aguda de un trastorno subyacente (intentos de suicidio, descompensaciones psicóticas, conductas heteroagresivas o altamente discapacitantes...); e incluso puede entenderse, en un sentido más amplio, como un momento de tránsito vital.

La intervención en crisis se centra en la acción de un equipo multidisciplinario, y las pautas esenciales según el modelo orientado al estrés serán:

- La ayuda ha de ser inmediata.

- Muy importante es proporcionar asistencia allí donde se produjo la crisis. De aquí la importancia de las visitas domiciliarias.

- Ofertar una continua disponibilidad.

- El enfoque se centrará en el problema y situación actual.

- Procurar que el individuo se mantenga en la realidad, evitando de esta manera que las negaciones o evasiones puedan conducir a soluciones desadaptativas neuróticas, o incluso a reacciones psicóticas posteriores.

- Procurar mantener la integridad del grupo familiar y estimular sus mecanismos positivos de afrontamiento del estrés.

Así, los tres objetivos principales de la intervención en crisis serán:

1) Definir la naturaleza del problema.

2) Implicar al sujeto y su entorno sociofamiliar, si es posible, en la búsqueda de soluciones al conflicto, asegurando a través de un número limitado de sesiones la readaptación del individuo/os en crisis.

3) Valorar la necesidad de un tratamiento individual/familiar más a largo plazo si fuese necesario.

En muchos casos la intervención en crisis ya solventa el problema, pero en otros sólo es el prólogo de un tratamiento posterior. Las técnicas utilizadas en este tipo de intervenciones son variadas, predominando las técnicas conductuales, las intervenciones sistémicas familiares, la psicoterapia de apoyo y las psicoterapias breves de inspiración psicodinámica aplicadas a la intervención en crisis.
En última instancia, la filosofía de la intervención en crisis es la de la prevención secundaria interviniendo sobre el individuo, la familia y el entorno, evaluando precozmente el conflicto, aplicando técnicas terapéuticas específicas y evitando así el desarrollo de complicaciones posteriores o la cronificación.

El individuo en crisis está incluido en un contexto familiar y social, por lo que el abordaje ha de ser global. Es necesario conocer la dinámica y estructura de la familia, su actitud hacia el miembro designado como paciente, su manera de afrontar crisis anteriores y los posibles factores de cambio o de conflicto que hayan podido ocurrir. Durante años la atención de los profesionales sanitarios se ha focalizado en las familias de pacientes mentales crónicos (repercusión de la enfermedad mental sobre el grupo familiar, influencia de la familia sobre el curso y evolución de la enfermedad, desarrollo de programas psicoeducativos, etc.), en detrimento de las situaciones agudas, en las cuales lo más frecuente es que la atención se centre en el paciente, obviándose la repercusión de la crisis sobre la familia.

La descompensación familiar puede considerarse desde un doble óptica: Cuando es toda la familia la que experimenta un estrés vital, no habiendo un único paciente identificado (por ejemplo, en el caso de la muerte de un familiar). Cuando, aún habiendo un paciente identificado, toda la familia participa del malestar y sufrimiento del individuo en crisis (o de las consecuencias de su actos); e incluso puede ocurrir que los problemas de este reflejen los de la familia. Así puede suceder que la inminencia de un cambio en la familia, la alteración en la comunicación o la aparición de conflictos entre sus miembros, suponga una ruptura del equilibrio intrafamiliar y la descompensación psicológica de uno de los miembros, más vulnerable en esos momentos. En ambos casos, y siempre que acuda la familia en una situación de urgencia, ha de incluirse a esta en la entrevista. Por varias razones: porque es una fuente de información primordial acerca de la historia del paciente y de la familia misma y porque es necesario ofertarles un espacio en el que puedan formular preguntas, expresar sus preocupaciones, miedos, sentimientos de culpa, de impotencia, etc. 
En una situación de urgencia las familias experimentan un gran estrés, que puede aumentar si se sienten ignorados o poco apoyados. En estas condiciones las actitudes pueden variar desde el enfado, irritabilidad, hostilidad franca hasta nula colaboración. Con la primera toma de contacto se intenta por una parte aliviar la ansiedad del grupo familiar ante una emergencia; y por otra parte, analizar la dinámica familiar así como su actitud hacia el paciente. Porque es necesario valorar si los problemas del paciente son producto de un conflicto intrapersonal, o son un reflejo de los de la familia. Si fuera así, el profesional debiera plantearse la conveniencia de integrar la familia en el tratamiento.

Por ello el terapeuta debe analizar paralelamente:

- Dinámica del grupo familiar. Grado de interacción, de comunicación, de vinculación afectiva, de individuación, asunción de roles de los miembros y subsistemas parental y filial.

- Estructura familiar. Aparte de valorar la estructura jerárquica, útil para orientar cada caso según sus necesidades y poder diferenciar entre un grupo familiar de estructura neurótica y un grupo familiar de estructura psicótica.

Se parte de un concepto de la familia como unidad de salud y enfermedad y permite, por tanto, objetivarla también como unidad de tratamiento. Los individuos y las familias que afrontan una crisis están más motivados para explorar los conflictos que la han podido originar, así como sus posibles soluciones, adoptando también actitud más flexible. La intervención sobre la familia en muchos casos es una alternativa primordial y fundamental, y en otros casos es una ayuda para solventar la situación.

En el grupo familiar de estructura neurótica los roles son flexibles, el que hace el rol de enfermo puede pasar al de sano y además no suele haber un único miembro designado como enfermo. Hay rivalidad entre los miembros aunque no envidia. Los cambios no se viven como amenazantes, no significan peligro de pérdida. Se buscará el apoyo y la implicación de la familia, aunque en última instancia el abordaje se centre directamente en el paciente.
En el grupo familiar de estructura psicótica, no significa necesariamente que sus miembros sean psicóticos, sino sus interrelaciones. Los roles son rígidos, inflexibles. El miembro designado como enfermo generalmente es único, y la enfermedad puede suponer la válvula de escape de la familia, donde se proyectan aspectos rechazados de cada uno. Los vínculos son de características simbióticas y las relaciones sadomasoquistas. Se viven los cambios como amenazantes y peligrosos, tendiendo a boicotearlos. En estos casos es toda la familia la que realmente se descompensa, y lo más idóneo es la asistencia terapéutica a todo el grupo de la forma más precoz posible, para evitar caer sistemáticamente en la hospitalización.

\section{Indicaciones para tratar a la familia:}

Es necesario abordar el tratamiento de la familia en las siguientes situaciones:

- Como ya se ha señalado con anterioridad, la descompensación familiar puede ocurrir cuando todos los miembros experimentan un trauma; por ejemplo, pérdida súbita de un ser querido, diagnóstico de una grave enfermedad a uno de los miembros, etc. Aunque en estos casos puede suceder que se designe a uno de los miembros como el necesitado de asistencia, lo indicado será el tratamiento de todos.

- También estará indicado, si se detectan conductas desadaptatívas repetitivas en la familia que puedan contribuir a la descompensación del miembro referido como paciente. Es decir, si los problemas de interacción pesan más que el conflicto individual.

- Cuando la familia está muy implicada en el problema del paciente, como consecuencia de interrelaciones patológicas, simbióticas.

- Cuando hay una historia de terapia individual de larga evolución y poco satisfactoria, conviene considerar la idoneidad de una terapia familiar dirigida a modificar el contexto familiar del sujeto. Cuando la familia obstaculiza el tratamiento individual por varios motivos (prejuicios, falsas creencias, desinformación, enfermedad como vía de escape de conflictos familiares...). 


\section{Estrategias para implicar a la familia}

Desde el primer contacto se debe desterrar cualquier implicación de responsabilidad o de contribución decisiva de la familia en el origen de la enfermedad, y trabajar con ellos para hacerles corresponsables de la asistencia terapéutica individual. Esto supone, la "utilización" de la familia como un recurso terapéutico.

En la práctica, la entrevista con la familia supone:

$1^{\mathrm{o}}$ Escuchar. Darles la oportunidad para que expongan sus puntos de vista, sus preocupaciones y temores, para que formulen preguntas.Las intervenciones del profesional en esta primera fase deben estar dirigidas a generar un clima de confianza y así disminuir la tensión, mostrando una actitud empática y comprensiva.

$2^{\circ}$ Intentar enfocar la crisis desde la perspectiva familiar. Averiguar cómo definen ellos el problema y cómo les afecta a cada uno, confrontando los diferentes puntos de vista. Ver qué factores creen que ha podido desencadenarlo, si ha habido otras crisis en el pasado y cómo las afrontaron, si ha habido o se prevé algún cambio en la estructura familiar. También indagar qué cree cada uno que puede hacer para solucionar el problema, o cómo piensan que se les puede ayudar. A medida que va transcurriendo esta fase, es importante subrayar las cualidades positivas que posea la familia, para disminuir la prevención defensiva y, a la vez, indicarles que las conductas conflictivas pueden formar parte de pautas de interacción familiar y de esta forma se facilita el cambio de atención desde el miembro identificado como paciente, hacia la relación familiar. Es fundamental que no se sugiera de manera prematura, ya que dicha interpretación puede conducir a un aumento de la resistencia y anular las posibilidades de cooperación de los miembros. El tratamiento familiar puede ser sugerido como una forma de ayudarles a que comprendan y afronten el problema.

$3^{\circ}$ Estimular la colaboración de la familia para solucionar el problema actual, e implicarla en el proceso terapéutico si es factible. No supone darles la impresión de que todos son pacientes, sino aclararles que se precisa su colaboración para que el problema se solvente de la forma más rápida y menos traumática posible. Por ejemplo, pueden: proporcionar apoyo y observación para evitar el ingreso hospitalario. Introducir modificaciones en el seno familiar, beneficiosas para el paciente. Colaborar cuando el paciente se niega a seguir las indicaciones médicas. Movilizarse en la búsqueda de recursos externos que puedan ser útiles. Hacer partícipe a la familia en la asistencia supone impedir el oscurecimiento de las relaciones intrafamiliares, aumentar su capacidad para afrontar futuras crisis, generar cambios adaptativos y evitar, en lo posible, la hospitalización y que la familia se reorganice para funcionar con el nuevo rol del paciente.

\section{BIBLIOGRAFÍA}

- Campoy A. (1980) Crisis e intervención en crisis. En: González de Rivera JL, Vela A, Arana J, eds. Manual de psiquiatría. Karpos. Madrid. pp 1191-1201.

- Caplan C. (1980) Principios de psiquiatría preventiva. Paidos. Buenos Aires.

- Comelles, J.M y Martínez Hernaez. (1993) Enfermedad, cultura y sociedad.: un ensayo sobre las relaciones entre la Antropología Social y la Medicina. Eudena. Madrid.

- Fernández Liria A. y Rodríguez Vega B. (2002) Intervención en crisis. Síntesis. Madrid.

- Fishel A, Gordon C. (1990) Tratamiento familiar en urgencias. En: Hyman SE, ed. Manual de urgencias psiquiátricas. 2.a ed. Salvat. Barcelona. pp 61-68.

- Gordon C. (1990) Intervención en crisis: ideas generales. En: Hyman S.E, ed. Manual de urgencias psiquiátricas. 2.a ed. Salvat. Barcelona. pp 39-46.

- Lazarus RS, Folkman S. (1986) El concepto de afrontamiento. En: Estrés y procesos cognitivos. Editorial Martínez-Roca S.A., Barcelona. pp 140-163.

- Leal, F. (2002) Comprender la enfermedad mental. Univ. de Murcia. Murcia.

- Llor B. y otros. (1998) Ciencias psicosociales aplicadas a la salud. Interamericana McGraw-Hill. Madrid.

- Mechanic D. (1962) The concept of illness behavior. J. Chron. London. pp 189-194.

- Parsons T. (1982) El sistema social. Alianza. Madrid.

- Pelicier N. (1989) La estrategia terapéutica de la crisis. Psicopatología; 9:23-25. 